\title{
THE MELLIN TRANSFORM AND SPECTRAL PROPERTIES OF TORIC VARIETIES
}

\author{
VICTOR GUILLEMIN AND ZUOQIN WANG
}

\begin{abstract}
In this article we apply results of $[\mathrm{W}]$ on the twisted Mellin transform to problems in toric geometry. In particular we use these results to describe the asymptotics of probability densities associated with the monomial eigenstates, $z^{k}, k \in \mathbb{Z}^{d}$, in Bargmann space and prove an "upstairs" version of the spectral density theorem of [BGU]. We also obtain for the $z^{k}$ 's, "upstairs" versions of the results of [STZ $]$ on distribution laws for eigenstates on toric varieties.
\end{abstract}

\section{INTRODUCTION}

Let $X$ be a compact Kähler manifold and $\mathbb{L} \rightarrow X$ a Hermitian line bundle whose curvature form is the negative of the Kähler form. For every positive integer $N$ let $\mathbb{L}^{N}$ be the $N^{\text {th }}$ tensor power of $\mathbb{L}$ and let $\pi_{N}$ be orthogonal projection

$$
\Gamma\left(\mathbb{L}^{N} ; X\right) \rightarrow \Gamma_{\text {hol }}\left(\mathbb{L}^{N} ; X\right) .
$$

The spectral measure, $\mu_{N}$, of the pair $\left(X, \mathbb{L}^{N}\right)$ is the measure defined by

$$
f \in C(X) \rightarrow \mu_{N}(f)=: \text { trace } \pi_{N} M_{f} \pi_{N}
$$

where $M_{f}$ is the operator "multiplication by $f$ ". It is known that as $N$ tends to infinity this measure has an asymptotic expansion in inverse powers of $N$ with distributional coefficients. (See, for instance, [BG].) However, the terms in this expansion have only been computed in a few special cases. One such case: $X$ a toric variety, was the topic of a recent article by Dan Burns, Alejandro Uribe and one of the authors of this paper, BGU]. One of the purposes of this note is to study the asymptotics of another interesting spectral measure associated with toric varieties. More explicitly, by Delzant's theorem every toric variety $X$ is the GIT quotient of $\mathbb{C}^{d}$ by a subtorus, $G$, of $T^{d}$, so $\Gamma_{\text {hol }}\left(\mathbb{L}^{N} ; X\right)$ has two descriptions: as holomorphic sections of $\mathbb{L}^{N}$ and as $G$-invariant holomorphic sections of $\left(\mathbb{L}_{\mathbb{C}^{d}}\right)^{N}$ where $\mathbb{L}_{\mathbb{C}^{d}}$ is the line bundle on $\mathbb{C}^{d}$ which gives rise by reduction to the line bundle, $\mathbb{L}$, on $X$. In the toric case this line bundle is the trivial bundle, $\mathbb{C}^{d} \times \mathbb{C}$; however the Hermitian inner product on it is the (non-trivial) Bargmann inner product. Namely for the trivializing section

$$
s: \mathbb{C}^{d} \rightarrow \mathbb{C}^{d} \times \mathbb{C}, \quad z \rightarrow(z, 1)
$$


of $\mathbb{L}_{\mathbb{C}^{d}}$

$$
\langle s, s\rangle=e^{-|z|^{2}} .
$$

Let $\mathbb{R}^{d}$ be the Lie algebra of $T^{d}, \mathfrak{g}$, the Lie algebra of $G$ and $e_{i}, i=1, \ldots, d$ the standard basis vectors of $\mathbb{R}^{d}$. From the inclusion, $\mathfrak{g} \rightarrow \mathbb{R}^{d}$, one gets a dual map $L:\left(\mathbb{R}^{d}\right)^{*} \rightarrow \mathfrak{g}^{*}$ and the weights of the representation of $G$ on $\mathbb{C}^{d}$ are just the vectors

$$
\alpha_{i}=L e_{i}^{*} .
$$

By Delzant's theorem every toric variety is obtained from $\mathbb{C}^{d}$ by reducing at a weight, $\alpha \in \mathbb{Z}_{G}^{*}$, and by the "quantization commutes with reduction" theorem $\Gamma_{\text {hol }}\left(\mathbb{L}^{N} ; X\right)$ is isomorphic as vector space to the space of $L^{2}$ holomorphic sections of $\left(\mathbb{L}_{\mathbb{C}^{d}}\right)^{N}$ which transform under $G$ according to the weight $\alpha$, in other words, the space:

$$
\Gamma_{\alpha}^{N}=\operatorname{span}\left\{z_{1}^{k_{1}} \ldots z_{d}^{k_{d}}, \sum k_{i} \alpha_{i}=N \alpha\right\} .
$$

Our first goal in this note is to study the spectral measure on $\mathbb{C}^{d}$ defined by

$$
\nu_{N}(f)=\operatorname{trace} \pi_{N} M_{f} \pi_{N}
$$

where $\pi_{N}$ is the orthogonal projection of the Bargmann space, $L^{2}\left(\mathbb{C}^{d}, e^{-N|z|^{2}} d z d \bar{z}\right)$, onto $\Gamma_{\alpha}^{N}$. This measure has many features in common with the measure, $\mu_{N}$, and in principle, one can be computed from the other. However, $\nu_{N}$ turns out to be much easier to compute. Moreover, the computation involves an object which is of considerable interest in itself: a "twisted" version of the classical Mellin transform.

The asymptotics of (1.5) is related via an Euler-Maclaurin formula to the asymptotics of the measure

$$
\frac{1}{c_{N, k}^{2}}\left|z^{k}\right|^{2} e^{-N|z|^{2}} d z d \bar{z}
$$

where the $c_{N, k}$ is chosen to make this a probability measure; and the second goal of this paper will be to study this asymptotics in detail, or, equivalently, to study the asymptotics of the integral

$$
\frac{1}{c_{N, k}^{2}} \int\left|z^{k}\right|^{2} e^{-N|z|^{2}} f d z d \bar{z}
$$

for $f \in \mathcal{C}_{0}^{\infty}\left(\mathbb{C}^{d}\right)$ and $k \in N \Delta$ as $N$ tends to infinity. The measure (1.6) can be thought of as the probability measure

$$
\left\langle s_{k}, s_{k}\right\rangle d z d \bar{z}
$$

associated with the "quantum state"

$$
s_{k}=\frac{1}{c_{N, k}} z^{k} \in \mathbb{L}_{\mathbb{C}^{d}}^{N}
$$


in Bargmann space, and our third goal in this paper will be to study the probability distribution on the real line associated with this quantum state:

$$
\sigma_{N, k}([t, \infty))=\operatorname{Vol}\left\{z \in \mathbb{C}^{d} \mid\left\langle s_{k}, s_{k}\right\rangle(z) \geq t\right\}
$$

The analogue of the distribution (1.8) for holomorphic sections of the line bundle, $\mathbb{L}$, "downstairs" on the toric variety, $X$, was studied in detail by Shiffman-TateZelditch in [STZ], and we will show that the distribution (1.8) has the same universal rescaling properties as theirs. (In fact these properties are much more transparently exhibited in this "upstairs" picture.)

Finally in the last section of this paper we will say a few words about how these "upstairs" and "downstairs" picture are related via some general results in GIT theory.

\section{The twisted Mellin transform}

For a bounded $\mathcal{C}^{\infty}$ function on $\mathbb{R}^{d}$ we define its " $N$-twisted" Mellin transform to be the quotient:

$$
A_{N} f(x)=\frac{\int_{\mathbb{R}_{+}^{d}} e^{N\left(\sum x_{i} \log y_{i}-y_{i}\right)} f(y) d y}{\int_{\mathbb{R}_{+}^{d}} e^{N\left(\sum x_{i} \log y_{i}-y_{i}\right)} d y} .
$$

For properties of this transform and the relation of (2.1) to the usual Mellin transform see $[\mathrm{W}]$. The main property we will need here is the following asymptotic expansion

$$
A_{N} f(x) \sim \sum_{\alpha} N^{-|\alpha|} f^{(\alpha)}(x) g_{\alpha}(N x)
$$

where

$$
g_{\alpha}(x)=g_{\alpha_{1}}\left(x_{1}\right) \ldots g_{\alpha_{d}}\left(x_{d}\right)
$$

and

$$
g_{k}(s)=\frac{1}{k !} \sum_{0 \leq \ell \leq k}(-1)^{\ell}\left(\begin{array}{l}
k \\
\ell
\end{array}\right) s^{\ell} s^{(k-\ell)}
$$

$s^{(m)}$ being the Hardy function

$$
s^{(m)}=(s+m) \ldots(s+1) .
$$

The sequence of functions $g_{k}(s)$ also has a nice generating function description in term of identity

$$
\sum_{r} g_{k}(s) x^{k}=\frac{e^{-s x}}{(1-x)^{1+s}}
$$


That (2.2) is in fact an asymptotic expansion follows from one of the main results of $[\mathrm{W}]$.

Theorem 2.1. The $k^{\text {th }}$ degree polynomial 2.4) is in fact a polynomial of degree $\left[\frac{k}{2}\right]$.

Thus the $\alpha^{\text {th }}$ summand in (2.2) is of order $N^{-\frac{|\alpha|}{2}}$.

We will now show how this asymptotics is related to the asymptotics of the measure (1.5). Let $\Delta$ be the convex polytope

$$
\left\{x \in \mathbb{R}_{+}^{d}, \sum x_{i} \alpha_{i}=\alpha\right\} .
$$

Then the functions, $z^{k}, k \in N \Delta \cap \mathbb{Z}^{d}$, are an orthogonal basis of $\Gamma_{\alpha}^{N}$ and the functions

$$
\frac{1}{c_{N, k}} z^{k}
$$

with

$$
c_{N, k}=\left(\int_{\mathbb{C}^{d}}\left|z^{k}\right|^{2} e^{-N|z|^{2}} d z d \bar{z}\right)^{\frac{1}{2}}
$$

are an orthogonal basis of $\Gamma_{\alpha}^{N}$. Hence the trace of $\pi_{N} M_{f} \pi_{N}$ is equal to the sum over $k \in N \Delta \cap \mathbb{Z}^{d}$ of

$$
\frac{\int\left|z_{1}\right|^{2 k_{1}} \ldots\left|z_{d}\right|^{2 k_{d}} e^{-N|z|^{2}} f(z) d z d \bar{z}}{\int\left|z_{1}\right|^{2 k_{1}} \ldots\left|z_{d}\right|^{2 k_{d}} e^{-N|z|^{2}} d z d \bar{z}}
$$

Now note that $\nu_{N}$ is $T^{d}$-invariant, so to compute $\nu_{N}(f)$ it suffices to compute $\nu_{N}(f)$ for functions which are themselves $T^{d}$-invariant, i.e., functions of the form $f\left(\left|z_{1}\right|^{2}, \ldots,\left|z_{d}\right|^{2}\right)$ where $f\left(x_{1}, \ldots, x_{n}\right)$ is a bounded $\mathcal{C}^{\infty}$ function on $\mathbb{R}^{d}$. However for such functions, (2.10) becomes

$$
\frac{\int_{\mathbb{R}_{+}^{d}} x_{1}^{k_{1}} \ldots x_{d}^{k_{d}} e^{-N\left(x_{1}+\cdots+x_{d}\right)} f(x) d x}{\int_{\mathbb{R}_{+}^{d}} x_{1}^{k_{1}} \ldots x_{d}^{k_{d}} e^{-N\left(x_{1}+\cdots+x_{d}\right)} d x} .
$$

This shows that the integral (1.7) is just the twisted Mellin transform, $A_{N}(f)(x)$ evaluated at $x=\frac{k}{N}$, and hence gives us for this integral the asymptotic expansion (2.2). Moreover, by summing (1.7) over the lattice points, $k \in N \Delta \cap \mathbb{Z}^{d}$ we get for the spectral measure, $\nu_{N}$, the formula

$$
\nu_{N}(f)=\sum_{k \in N \Delta \cap \mathbb{Z}^{d}} A_{N} f\left(\frac{k}{N}\right) .
$$

In the next section we will get an asymptotic expansion for $\nu_{N}(f)$ by interpreting the sum on the right as a Riemann sum and combining the formula (2.2) with an Euler-Maclaurin formula for Riemann sums over convex polytopes. 


\section{Riemann sums}

Let $\Delta \subseteq \mathbb{R}^{n}$ be an $n$-dimensional convex polytope. By elementary calculus the Riemann integral of a function $f \in \mathcal{C}^{\infty}(\Delta)$ :

$$
\int_{\Delta} f(x) d x
$$

is approximated by the Riemann sum

$$
\frac{1}{N^{n}} \sum_{k \in N \Delta \cap \mathbb{Z}^{n}} f\left(\frac{k}{N}\right)
$$

up to an error term of order $O\left(N^{-1}\right)$.

Recently Guillemin and Sternberg showed that if $\Delta$ is a lattice polytope, i.e., if its vertices are lattice points, then this $O\left(N^{-1}\right)$ can be replaced by an asymptotic series in inverse powers of $N$. In particular for polytopes associated with toric varieties (such as the polytope (2.7)) the terms in this series can be explicitly computed by the following method.

Enumerate the facets of $\Delta$, and for the $i^{\text {th }}$ facet let $u_{i} \in \mathbb{Z}^{n}$ be a primitive lattice vector which is perpendicular to this facet and points "outward" from $\Delta$ into $\mathbb{R}^{n}$. Then $\Delta$ can be defined by a set of inequalities

$$
\left\langle u_{i}, x\right\rangle \leq c_{i}, \quad i=1, \ldots, r
$$

where $r$ is the number of facets. Let $\Delta_{h}$ be the polytope

$$
\left\langle u_{i}, x\right\rangle \leq c_{i}+h_{i}, \quad i=1, \ldots, r .
$$

Then for $f \in \mathcal{C}^{\infty}\left(\mathbb{R}^{n}\right)$

$$
\frac{1}{N^{n}} \sum_{k \in \mathbb{Z}^{n} \cap N \Delta} f\left(\frac{k}{N}\right) \sim\left(\tau\left(\frac{1}{N} \frac{\partial}{\partial h}\right) \int_{\Delta_{h}} f(x) d x\right)(h=0)
$$

where

$$
\tau\left(w_{1}, \ldots, w_{r}\right)=\prod_{i=1}^{r} \frac{w_{i}}{1-e^{-w_{i}}}
$$

and $\tau\left(\frac{1}{N} \frac{\partial}{\partial h}\right)$ is the operator obtained from (3.4) by making the substitution $w_{i} \rightarrow$ $\frac{1}{N} \frac{\partial}{\partial h_{i}}$. (The leading term on the right hand side of (3.3) is the integral of $f$ over $\Delta$ and the higher order terms are the asymptotic series in inverse powers of $N$ that we just alluded to.)

Now notice that if we divide (2.12) by $N^{n}$ the right hand side is exactly a Riemann sum of the form above. Hence if we replace $A_{N} f$ by the series (2.2) and apply (3.3) to each summand we get an asymptotic expansion of $\nu_{N}(f)$ in inverse powers of $N$ in which the summands can be read off from the summands on the 
right hand side of (3.3). (For a more detailed description of these summands see $\S 3$ of [GS06]).

\section{The Nonrescaled Distribution Law}

We turn now to the third topic of this paper: the asymptotics of the probability distribution (1.8). Suppose $k=N a$ with $a \in \Delta$. We begin by observing that

$$
\left\|z^{k}\right\|_{N}^{2}=\int_{\mathbb{C}^{d}}\left\langle z^{k}, z^{k}\right\rangle_{N} d z d \bar{z}=\left(\frac{\pi}{N}\right)^{d} \prod_{i} \frac{\left(k_{i}\right) !}{N^{k_{i}}},
$$

and hence

$$
\left\langle s_{k}, s_{k}\right\rangle_{N}=\left(\frac{N}{\pi}\right)^{d} \frac{N^{|k|}}{k !}\left|z^{k}\right|^{2} e^{-N|z|^{2}} .
$$

We first assume that $k=\left(k_{1}, \cdots, k_{d}\right)$ with $k_{i}>0$ for all $1 \leq i \leq d$, and observe that $\sigma_{N, k}([t, \infty))$ is the volume of the region in $\mathbb{C}^{d}$

$$
\left|z^{k}\right|^{2} e^{-N|z|^{2}}>\left(\frac{\pi}{N}\right)^{d} \frac{k !}{N^{|k|}} t,
$$

or, with $a=\frac{k}{N}$, the region

$$
\left|z^{a}\right|^{2} e^{-|z|^{2}}>\left(\left(\frac{\pi}{N}\right)^{d} \frac{k !}{N^{|k|}} t\right)^{1 / N}
$$

By Stirling's formula,

$$
k_{i} !=\sqrt{2 \pi k_{i}}\left(\frac{k_{i}}{e}\right)^{k_{i}}\left(1+O\left(\frac{1}{N}\right)\right),
$$

so the right hand side of (4.3) is equal to

$$
\lambda_{N}=\left(\pi^{d} N^{-d / 2} t \prod_{i}\left(2 \pi a_{i}\right)^{1 / 2}\right)^{1 / N}\left(\frac{a}{e}\right)^{a}\left(1+O\left(\frac{1}{N^{2}}\right)\right) .
$$

Thus if we set $\left|z_{i}\right|^{2}=r_{i}$ and let $f(r)$ be the function

$$
f(r)=\sum_{i=1}^{d}\left(a_{i} \log r_{i}-r_{i}\right),
$$

the inequality (4.3) becomes

$$
f(r) \geq \log \lambda_{N}=\sum_{i}\left(a_{i} \log a_{i}-a_{i}\right)-\frac{d}{2 N} \log N+\frac{\log t+\gamma}{N}+O\left(\frac{1}{N^{2}}\right),
$$

where

$$
\gamma=\log \left(\pi^{d} \prod_{i}\left(2 \pi a_{i}\right)^{1 / 2}\right)
$$


We now note that $f(r)$ has a unique maximum at $r=a$ and that in a neighborhood of this maximum,

$$
f(r)=\sum_{i}\left(a_{i} \log a_{i}-a_{i}-\frac{1}{2 a_{i}}\left(r_{i}-a_{i}\right)^{2}\right)+\cdots .
$$

Hence for $N$ large (ignoring terms in $N$ of order $O\left(\frac{1}{N}\right)$ ) (4.3) reduces to

$$
\sum_{i} \frac{1}{2 a_{i}}\left(r_{i}-a_{i}\right)^{2} \leq \frac{d}{2 N} \log N
$$

or, since $r_{i}=\left|z_{i}\right|^{2}$,

$$
\sum_{i} \frac{1}{2 a_{i}}\left(\left|z_{i}\right|^{2}-a_{i}\right)^{2} \leq \frac{d}{2 N} \log N+O\left(\frac{1}{N}\right) .
$$

To compute the volume of this set to the leading order, we first note that the volume of the ellipsoid

$$
\sum_{i=1}^{d} \frac{1}{2 a_{i}} x_{i}^{2} \leq \varepsilon
$$

in $\mathbb{R}^{d}$ is

$$
\gamma_{d}\left(\prod 2 a_{i}\right)^{1 / 2} \varepsilon^{d / 2}
$$

where $\gamma_{d}$ is the volume of the unit $d$-ball. Now consider the map

$$
g: \mathbb{R}_{+}^{d} \rightarrow \mathbb{R}^{d}, \quad s_{i} \mapsto x_{i}=s_{i}^{2}-a_{i} .
$$

The pre-image of the region (4.7) with respect to this map is the set

$$
\sum \frac{1}{2 a_{i}}\left(s_{i}^{2}-a_{i}\right)^{2} \leq \varepsilon .
$$

If $s$ is a point in this set, then $s_{i}=\sqrt{a_{i}}+O\left(\varepsilon^{1 / 4}\right)$, so

$$
\operatorname{det}(D g(s))=\prod_{i}\left(2 s_{i}\right)=2^{d} \prod_{i} \sqrt{a_{i}},
$$

and thus by (4.8) the volume of the region (4.9) is equal, modulo $O\left(\varepsilon^{1 / 4}\right)$, to

$$
\gamma_{d}\left(\frac{\varepsilon}{2}\right)^{d / 2}
$$

Finally note that the region (4.6) is, with $\varepsilon=\frac{d}{2 N} \log N$, the pre-image of the region (4.9) with respect to the torus fibration, $s_{i}=\left|z_{i}\right|$. Since each torus fiber has volume $\prod\left(2 \pi s_{i}\right)$ and $s_{i}=\sqrt{a_{i}}+O\left(\varepsilon^{1 / 4}\right)$, the total volume of the region (4.6) is equal modulo a factor of $1+O\left(\varepsilon^{1 / 4}\right)$ to

$$
(2 \pi)^{d} \gamma_{d}\left(\prod_{i} \frac{a_{i} \varepsilon}{2}\right)^{1 / 2},
$$


and hence by substituting $\frac{d \log N}{2 N}$ for $\varepsilon$ we arrive finally at the asymptotic formula

$$
\sigma_{N, k}([t, \infty)) \sim \pi^{d} \gamma_{d} \prod_{i}\left(a_{i} \frac{d}{N} \log N\right)^{1 / 2} .
$$

Remark 4.1. More generally suppose $k=\left(k_{1}, \cdots, k_{l}, 0, \cdots, 0\right)$ with $k_{i}>0$ for $1 \leq i \leq l$, then $\sigma_{N, k}([t, \infty))$ is, to its leading order, equal to the volume of the region

$$
\sum_{i=1}^{l} \frac{1}{2 a_{i}}\left(\left|z_{i}\right|^{2}-a_{i}\right)^{2}+\sum_{i=l+1}^{d}\left|z_{i}\right|^{2} \leq \frac{d}{2 N} \log N
$$

To compute the volume of this set, we regard it as the pre-image of the $l$-torus fibration over the $2 d-l$ dimensional ellipsoid

$$
\sum_{i=1}^{l} \frac{1}{2 a_{i}}\left(s_{i}^{2}-a_{i}\right)^{2}+\sum_{i=l+1}^{d}\left(x_{i}^{2}+y_{i}^{2}\right) \leq \frac{d}{2 N} \log N,
$$

and by the same argument as above, get

$$
\sigma_{N, k}([t, \infty)) \sim 2^{l-d} \pi^{l} \gamma_{2 d-l}\left(\frac{d \log N}{N}\right)^{d-\frac{l}{2}} \prod_{i}\left(a_{i}\right)^{1 / 2} .
$$

\section{Rescaled Distribution Laws}

For simplicity we assume all $k_{i}$ are positive. From (4.3), (4.4) and (4.5) we have

$$
\sum_{i=1}^{d} \frac{1}{2 a_{i}}\left(\left|z_{i}\right|^{2}-a_{i}\right)^{2} \leq \varepsilon_{N}
$$

where

$$
\varepsilon_{N}=\frac{d}{2 N} \log N-\frac{\log t+\gamma}{N}+o\left(\frac{1}{N}\right)
$$

Thus the $t$ term gets absorbed in the $O\left(\frac{1}{N}\right)$ and doesn't affect the leading asymptotics of $\sigma_{N}([t, \infty))$. However, we can remedy this problem by rescaling techniques.

The first choice of rescaling is to eliminate the leading term $\frac{d}{2 N} \log N$. To do so, we replace $t$ by $N^{d / 2} t$. Then

$$
\varepsilon_{N}=(-\log t-\gamma) \frac{1}{N}+o\left(\frac{1}{N}\right)
$$

and the computations in the last section show that this rescaled version of $\sigma_{N, k}([t, \infty))$ satisfies (4.9) with $\varepsilon=\varepsilon_{N}$ given by (5.4) and hence depends in an interesting way on $t$. (One proviso, however, is that $\log t$ has to be smaller than $-\gamma$.) 
There are also many other interesting choices of rescalings: we may rescale $t$ such that the term containing $\log t$ dominate other terms. For example, we may replace $t$ by $e^{-N^{\alpha}(\log N)^{\beta} t}$, where $0<\alpha<1$ or $\alpha=0, \beta>1$. In this case

$$
\varepsilon_{N}=N^{\alpha-1}(\log N)^{\beta} t+O\left(\frac{\log N}{N}\right) .
$$

We may also replace $t$ by $N^{-t}$, which is the extreme case $\alpha=0, \beta=1$ above, then

$$
\varepsilon_{N}=\frac{d+2 t}{N} \log N+O\left(\frac{1}{N}\right)
$$

\section{6. "Upstairs" versus "DOWnstairs"}

In GIT theory one is given a projective variety, $M$, a positive line bundle, $\mathbb{L} \rightarrow M$, and an action of an algebraic group, $G_{\mathbb{C}}$, on the pair $(M, \mathbb{L})$; and one's goal is to make sense of the quotients, $M / G_{\mathbb{C}}$ and $\mathbb{L} / G_{\mathbb{C}}$. Unfortunately $M / G_{\mathbb{C}}$ can be a fairly pathological object, even as a topological space (e.g. it can be non-Hausdorff); however, $M$ contains a $G_{\mathbb{C}}$ invariant Zariski open set, $M_{\text {stable }}$ for which the quotient

$$
M_{\text {red }}=M_{\text {stable }} / G_{\mathbb{C}}
$$

is a quasi-projective variety. Moreover if $\mathbb{L}_{\text {stable }}$ is the restriction of $\mathbb{L}$ to $M_{\text {stable }}$ one gets a quotient line bundle

$$
\mathbb{L}_{\text {red }}=\mathbb{L}_{\text {stable }} / G_{\mathbb{C}}
$$

on $M_{r e d}$, and modulo some Kaehlerizability assumptions one can equip $\mathbb{L}$ and $\mathbb{L}_{r e d}$ with natural Hermitian inner products, $\langle\cdot, \cdot\rangle$ and $\langle\cdot, \cdot\rangle_{\text {red }}$ which are related by an identity

$$
\pi^{*}\langle\cdot, \cdot\rangle_{\text {red }}=e^{\psi}\langle\cdot, \cdot\rangle
$$

where $\pi$ is the projection of $M_{\text {stable }}$ onto $M_{r e d}$ and $\psi$ is a $\mathcal{C}^{\infty}$ function called the stability function. (See [GS82, BGU], HK], L] etc.)

In the case of toric varieties $M$ is $\mathbb{C} P^{d-1}$ (or more simply its de-projectivization, $\left.\mathbb{C}^{d}\right), G_{\mathbb{C}}$ is a complex torus and the action of $G_{\mathbb{C}}$ on $\mathbb{C}^{d}$ is a linear action; and in the toric variety case the stability function turns out to be given by a fairly simple formula: If $X=M_{r e d}$ is the reduction of $\mathbb{C}^{d}$ at a weight, $\alpha$, of $G, \psi$ is of the form

$$
\psi=-|z|^{2}+f,
$$

where $f$ transforms under the action of $G_{\mathbb{R}}$ according to the character, $e^{\alpha}$. From this description of $\psi$ one can conclude quite a bit about the asymptotic behavior of norm-squares of holomorphic sections of $\mathbb{L}_{X}^{N}$ from the "upstairs picture" on $\mathbb{C}^{d}$ that we've described in this paper. In particular one can obtain some of the results of Shiffman-Tate-Zelditch that we mentioned in the introduction by combining results about "moments of measures" proved by them in $\S 4.1$ of their paper with asymptotic 
properties of $e^{N \psi}$ and results of $\S 5$ above. We will give an account of this stability theory for toric varieties in $\mathrm{GW}$.

\section{REFERENCES}

[BG] L. Boutet de Monvel and V. Guillemin, The spectral theory of Toeplitz operators, Annals of Math. Studies 99, Princeton U. Press, Princeton, NJ, 1981.

[BGU] D. Burns, V. Guillemin and A. Uribe, "The Spectral Density Functions of a Toric Variety", math.SP/0706.3039.

[G] V. Guillemin, Moment Maps and Combinatorial Invariants of Hamiltonian Tn-spaces, Progress in Mathematics 122, Birkhauser Boston, Boston, MA, 1994.

[GS82] V. Guillein and S. Sternberg, "Geometric quantization and multiplicities of group representations", Invent. Math. 67, 515-538, 1982.

[GS06] V. Guillemin and S. Sternberg, "Riemann Sums over Polytopes", Ann. Inst. Fourier (to appear)

[GW] V. Guillemin and Z. Wang, "Stability functions" (In preparation)

$[\mathrm{HK}]$ B. Hall and W. Kirwin, "Unitarity in "quantization commutes with reduction", math.SG/0610005 4, to appear in Comm. Math. Phys.

[L] H. Li, "Singular unitarity in quantization commutes with reduction", math.SG/0706.1471v1.

[STZ] B. Shiffman, T. Tate and S. Zelditch, "Distribution Laws for Integrable Eigenfunctions", Ann. Inst. Fourier (Grenoble), 54 (2004), no. 5, 1497C1546.

[W] Wang, Z. "The Twisted Mellin Transform", math.CO/0706.2642.

Department of Mathematics, Mit, Cambridge, MA 02139, USA

E-mail address: vwg@math.mit.edu

Department of Mathematics, Mit, Cambridge, MA 02139, USA

E-mail address: wangzq@math.mit.edu 